\title{
Disparate roles of zinc in chemical hypoxia-induced neuronal death
}

\author{
Sujeong Kim ${ }^{1 \dagger}$, Jung-Woo Seo ${ }^{1 \dagger}$, Shin Bi Oh ${ }^{1}$, So Hee Kim ${ }^{1}$, Inki Kim ${ }^{1}$, Nayoung Suh ${ }^{1}$ and Joo-Yong Lee ${ }^{1,2 *}$ \\ Asan Institute for Life Sciences, Asan Medical Center, Seoul, South Korea \\ ${ }^{2}$ Department of Neurology, University of Ulsan College of Medicine, Seoul, South Korea
}

\section{Edited by:}

Qi Yuan, Memorial University,

Canada

\section{Reviewed by:}

Hongyu Sun, University of

Pennsylvania, USA

Xin Wang, Stanford University and

Howard Hughes Medical Institute,

USA

\section{*Correspondence:}

Joo-Yong Lee, Asan Institute for Life Sciences, Asan Medical Center,

Seoul 138-736, South Korea

e-mail: jlee@amc.seoul.kr

${ }^{\dagger}$ These authors have contributed equally to this work.
Accumulating evidence has provided a causative role of zinc $\left(\mathrm{Zn}^{2+}\right)$ in neuronal death following ischemic brain injury. Using a hypoxia model of primary cultured cortical neurons with hypoxia-inducing chemicals, cobalt chloride ( $\left.1 \mathrm{mM} \mathrm{CoCl}_{2}\right)$, deferoxamine ( $3 \mathrm{mM} \mathrm{DFX),}$ and sodium azide ( $2 \mathrm{mM} \mathrm{NaN}_{3}$ ), we evaluated whether $\mathrm{Zn}^{2+}$ is involved in hypoxic neuronal death. The hypoxic chemicals rapidly elicited intracellular $\mathrm{Zn}^{2+}$ release/accumulation in viable neurons. The immediate addition of the $\mathrm{Zn}^{2+}$ chelator, CaEDTA or N,N, N'N'-tetrakis(2-pyridylmethyl) ethylenediamine (TPEN), prevented the intracellular $\mathrm{Zn}^{2+}$ load and $\mathrm{CoCl}_{2}-$ induced neuronal death, but neither 3 hour later $\mathrm{Zn}^{2+}$ chelation nor a non- $\mathrm{Zn}^{2+}$ chelator ZnEDTA $(1 \mathrm{mM})$ demonstrated any effects. However, neither CaEDTA nor TPEN rescued neurons from cell death following DFX- or $\mathrm{NaN}_{3}$-induced hypoxia, whereas ZnEDTA rendered them resistant to the hypoxic injury. Instead, the immediate supplementation of $\mathrm{Zn}^{2+}$ rescued DFX- and $\mathrm{NaN}_{3}$-induced neuronal death. The iron supplementation also afforded neuroprotection against DFX-induced hypoxic injury. Thus, although intracellular $\mathrm{Zn}^{2+}$ release/accumulation is common during chemical hypoxia, $\mathrm{Zn}^{2+}$ might differently influence the subsequent fate of neurons; it appears to play a neurotoxic or neuroprotective role depending on the hypoxic chemical used. These results also suggest that different hypoxic chemicals may induce neuronal death via distinct mechanisms.

Keywords: metal chelation, iron, delayed neuronal death, neuroprotection, brain injury

\section{INTRODUCTION}

Zinc $\left(\mathrm{Zn}^{2+}\right)$ contributes to neuronal injury according to various experimental models of excitotoxic brain injury (Sensi et al., 2011). Exposing cortical cultures to high levels of $\mathrm{Zn}^{2+}$ induces extensive neuronal and glial cell death (Choi et al., 1988). The intracellular release of $\mathrm{Zn}^{2+}$ subsequent to exposure to oxidative or nitrosative agents leads to neuronal degeneration in cultured neurons (Bossy-Wetzel et al., 2004; Hwang et al., 2008). In animal models of acute brain injury, including cerebral ischemia, epilepsy, and trauma, a large accumulation of $\mathrm{Zn}^{2+}$ occurs in degenerating neurons as demonstrated by the $\mathrm{Zn}^{2+}$-specific fluorescence dyes (Frederickson et al., 1988; Tønder et al., 1990; Koh et al., 1996; Lee et al., 2000; Suh et al., 2000). Intracellular $\mathrm{Zn}^{2+}$ release/accumulation obviously precedes neuronal death in these experimental models (Koh et al., 1996) since $\mathrm{Zn}^{2+}$ chelators, such as ethylenediaminetetraacetic acid (EDTA; Koh et al., 1996; Lee et al., 2000; Frederickson et al., 2002; Suh et al., 2004) and $\mathrm{N}, \mathrm{N}, \mathrm{N}^{\prime}, \mathrm{N}^{\prime}$,-tetrakis-(2-pyridylmethyl)ethylenediamine (TPEN; Bossy-Wetzel et al., 2004; Cho et al., 2010), intercept intracellular $\mathrm{Zn}^{2+}$ load to suppress neuronal death

Cerebral hypoxia develops when the brain suffers from oxygen shortage due to the blockage of blood flow, resulting in extensive neuronal death in selective vulnerable areas (Sharp and Bernaudin, 2004). Since the involvement of $\mathrm{Zn}^{2+}$ in neuronal death in the hippocampal CA1 area following transient global cerebral ischemia was reported (Koh et al., 1996), studies have suggested that excessive $\mathrm{Zn}^{2+}$ release/accumulation leads to neuronal injury after hypoxia/ischemia (Sensi et al., 2011). When mouse hippocampal slices are subjected to oxygen and glucose deprivation (OGD) - which is a typical experimental model of hypoxia/ischemia-intracellular $\mathrm{Zn}^{2+}$ becomes prominent in degenerating neurons, whereby the $\mathrm{Zn}^{2+}$ chelator CaEDTA attenuates both $\mathrm{Zn}^{2+}$ accumulation and neuronal death (Yin et al., 2002; Medvedeva et al., 2009). Similarly, hypobaric hypoxia causes $\mathrm{Zn}^{2+}$-mediated inflammation and apoptosis in neurons of the mouse hippocampus, which are also reversed by CaEDTA (Malairaman et al., 2014). Recent studies have provided that $\mathrm{Zn}^{2+}$ promotes hypoxic cell death by upregulating hypoxia-inducible transcription factor- $1 \alpha(\mathrm{HIF} 1 \alpha)$ via an activation of NADPH oxidase or poly(ADP-ribose) polymerase (PARP; Pan et al., 2013; Malairaman et al., 2014).

While the precise control of oxygen level is crucial to simulate hypoxic condition in cell culture, it is difficult, so various in vitro models of neuronal hypoxia have been provided containing OGD models. Some divalent cations such as cobalt $\left(\mathrm{Co}^{2+}\right)$, nickel $\left(\mathrm{Ni}^{2+}\right)$, and the iron-chelator deferoxamine (DFX), have been applicable to mimic hypoxic conditions in cultured cells because they activate hypoxic signals by stabilizing 
the expression of HIF1 $\alpha$ (Ho and Bunn, 1996). Sodium azide $\left(\mathrm{NaN}_{3}\right)$ and potassium cyanide $(\mathrm{KCN})$ are also potent inhibitors of cytochrome c oxidase (i.e., complex IV of the mitochondrial respiratory chain) to induce chemical hypoxia (Roemgens et al., 2011). However, although the hypoxic chemicals have helped us to understand the molecular events that underlie the hypoxic neuronal death, it remains unclear whether chemical hypoxia also involves $\mathrm{Zn}^{2+}$-mediated neuronal injury in cultured neurons.

In this study, we found that intracellular $\mathrm{Zn}^{2+}$ release/ accumulation occurs in primary neuronal cells shortly after exposure to $\mathrm{CoCl}_{2}$, DFX, or $\mathrm{NaN}_{3}$, whereas the effects of $\mathrm{Zn}^{2+}$ chelation on neuronal fate differ depending on the hypoxiainducing chemicals used. This study shows the disparate roles of $\mathrm{Zn}^{2+}$ in neuronal death following chemical hypoxia.

\section{MATERIALS AND METHODS PRIMARY CORTICAL NEURON CULTURES}

We used ICR mice in this study, in accordance with the Guidelines of the Asan Institute for Life Sciences for the Care and Use of Laboratory Animals. Cerebral cortical tissues were dissected from the brains of fetal ICR mice (Koatech, Pyeongtaek, Korea) at embryonic day E14, dissociated in $\mathrm{Ca}^{2+} / \mathrm{Mg}^{2+}$-free Hank's balanced salt solution (HBSS; Invitrogen, Carlsbad, CA, USA) containing $0.25 \%$ trypsin-EDTA (Invitrogen), and filtered through 40- $\mu \mathrm{m}$ nylon cell strainer (BD Biosciences, Durham, NC, USA). Cells were washed in Dulbecco's modified Eagle's medium (DMEM; Invitrogen) with 10\% fetal bovine serum (FBS; Gibco, Grand Island, NY, USA) and penicillin/streptomycin (Invitrogen), and resuspended in serum-free Neurobasal medium (Invitrogen) containing the B27 supplement (Invitrogen), L-glutamine (2 mM; Invitrogen) and penicillin/streptomycin. Cells were plated at a density of $5 \times 10^{5}-10^{6}$ cells/well on poly-L-lysine-coated well culture dishes and grown in a humidified $5 \% \mathrm{CO}_{2}$ incubator at $37^{\circ} \mathrm{C}$. Cultures were treated with cytosine arabinoside (Ara-C, 2 $\mu \mathrm{M}$; Sigma, St. Louis, MO, USA) for $24 \mathrm{~h}$ at 3 days in vitro (DIV3) to halt the growth of non-neuronal cells, and maintained in fresh Neurobasal medium with B27 until used in experiments between DIV10-11.

\section{INDUCTION OF CHEMICAL HYPOXIA}

All chemicals used in this study, except CoEDTA (TCI, Tokyo, Japan), were purchased from Sigma-Aldrich or Fluka (St. Louis, MO, USA).

To induce chemical hypoxia, cells were treated with $\mathrm{CoCl}_{2}$ (1 mM) (Fang et al., 2008; Zhang et al., 2011) or DFX (3 mM) (Almli et al., 2001; Guelman et al., 2004) for $2 \mathrm{~h}$, or $\mathrm{NaN}_{3}(2 \mathrm{mM}$ ) for $1 \mathrm{~h}$ (Garnier et al., 2003; Selvatici et al., 2009) in glucose-free MEM, and then the media was freshly replaced. To define the roles of the intracellular metals in neurons during chemical hypoxia, we added the metal chelator EDTA with various salts (CaEDTA, ZnEDTA, CoEDTA, or FeEDTA), or TPEN to the media at $10 \mathrm{~min}$ or $3 \mathrm{~h}$ after hypoxic chemical treatment.

\section{CELL VIABILITY ASSESSMENT}

Cell viability was determined using the 3-(4,5-dimethylthiazol2-yl)-2,5-diphenyltetrazolium bromide (MTT) assay (Stanciu et al., 2000; White et al., 2001). Cortical neurons were grown on poly-L-lysine coated 24 -well plates, and treated with MTT (final concentration, $0.5 \mathrm{mg} / \mathrm{mL}$ in culture media; Amresco, Solon, $\mathrm{OH}, \mathrm{USA}$ ) at $37^{\circ} \mathrm{C}$ for $2 \mathrm{~h}$. After culture medium was completely removed, the insoluble formazan crystals were dissolved in dimethyl sulfoxide (DMSO; $200 \mu \mathrm{L}$ ). The reaction products (in $100 \mu \mathrm{L}$ aliquots) were measured at $570 \mathrm{~nm}$ using a microplate reader (Synergy H1 Hybrid; BioTek Instruments, Winooski, VT, USA). All experiments were consisted of at least three independent repeats, and each experiment contained three parallel cultures. Duplicate measurements of MTT absorbance were performed for each sample. Resultantly, percentage of viable cells in drug-treated cultures was determined relative to vehicle-treated control cells.

In addition, neuronal cell death was visually detected by staining the nuclei with Hoechst 33342 or propidium iodide (PI). After the cells were incubated in the presence of Hoechst 33342 (10 $\mu \mathrm{g} / \mathrm{mL}$; Invitrogen) and PI (1 $\mu \mathrm{g} / \mathrm{mL}$; Sigma) for $15 \mathrm{~min}$, the fluorescent phenotypes of the nuclei were examined under a fluorescence inverted microscope (Axio Observer.Z1; Carl Zeiss, Göttingen, Germany) using a DAPI filter (beam splitter, $395 \mathrm{~nm}$; excitation, $365 \pm 50 \mathrm{~nm}$; emission, $445 \pm 50 \mathrm{~nm}$ ) and a Set20 filter (beam splitter, $560 \mathrm{~nm}$; excitation, $546 \pm 12 \mathrm{~nm}$; emission, 575-640 nm), respectively. PI-fluorescent red nuclei-containing neurons were considered dead or dying as the dye is excluded by viable cells.

\section{DETECTION AND MEASUREMENT OF INTRACELLULAR ZN ${ }^{2+}$}

To assess the levels of intracellular $\mathrm{Zn}^{2+}$, cells were incubated with $2 \mu \mathrm{M}$ FluoZin-3 AM (Kd for $\mathrm{Zn}^{2+}$, about $15 \mathrm{nM}$ ) (Molecular Probes, Eugene, OR, USA) for $30 \mathrm{~min}$ and washed with fresh culture medium (Gee et al., 2002). FluoZin-3 reactive cells were examined or photographed under consistent imaging conditions with an inverted fluorescence microscope (Axio Observer.Z1) using a FITC filter (beam splitter, 495 $\mathrm{nm}$; excitation, 450-490 nm; emission, 500-550 nm) equipped with self-adjusting lamps and an AxioCam digital camera (Carl Zeiss).

To quantify the level of intracellular $\mathrm{Zn}^{2+}$, we took the photographs (magnification, 100 $\times$ ) from three spots randomly selected from each culture well, and measured the mean intensity of FluoZin-3-fluorescence in neurons using ImagePro Plus software (Media Cybernetics, Silver Spring, MD, USA). After subtracting the background intensity (which was determined by assessing areas without cells), the average intensity of FluoZin-3-fluorescence per neuron was reported as the level of intracellular $\mathrm{Zn}^{2+}$.

\section{STATISTICAL ANALYSIS}

Values were expressed as the mean \pm standard errors of mean (SEM). Statistical comparisons were performed using one-way analysis of variance (ANOVA) followed by the post hoc StudentNewman-Keuls test using GraphPad InStat (GraphPad Software, La Jolla, CA, USA). $P$ values $<0.05$ were considered to indicate statistical significance. 


\section{RESULTS}

Since an early study implicated $\mathrm{Zn}^{2+}$ in neuronal death following transient global cerebral ischemia in rats (Koh et al., 1996), a large body of evidence has attributed excitotoxic neuronal injury to $\mathrm{Zn}^{2+}$ overload in neurons (Sensi et al., 2011). This is principally based on the proof-of-concept that intracellular $\mathrm{Zn}^{2+}$ overload occurs in degenerating neurons (correlation) before death (precedence), and that such pathological phenomena are eliminated when $\mathrm{Zn}^{2+}$ is chelated or removed (interference) (Koh et al., 1996). However, to our knowledge, there are a few study regarding that intracellular $\mathrm{Zn}^{2+}$ indeed takes part in hypoxic neuronal death, except experiments in which cerebral organ cultures (but not neuronal cells) were exclusively subjected to OGD conditions (Büchner et al., 2002; Yin et al., 2002; Miyawaki et al., 2004; Medvedeva et al., 2009). Thus, we first performed our current study to determine if $\mathrm{Zn}^{2+}$ release/accumulation occurs in association with neuronal death in primary neuronal cultures exposed to a hypoxic chemical, $\mathrm{CoCl}_{2}, \mathrm{DFX}$, or $\mathrm{NaN}_{3}$.

\section{INTRACELLULAR ZN ${ }^{2+}$ RELEASE/ACCUMULATION IN NEURONS EXPOSED TO HYPOXIC CHEMICALS}

Intracellular $\mathrm{Zn}^{2+}$ was detected using the $\mathrm{Zn}^{2+}$-specific fluorescent indicator FluoZin-3 (Gee et al., 2002), which reacted with $\mathrm{Zn}^{2+}$ to emit bright green fluorescence in cortical neurons $30 \mathrm{~min}$ after the addition of $200 \mu \mathrm{M} \mathrm{ZnCl}_{2}$ (Figures 1B,M). Three hours later, FluoZin-3-fluorescence was significantly attenuated in the $\mathrm{ZnCl}_{2}$-treated neuron cultures (Figures $\mathbf{1 C}, \mathbf{M}$ ).

Similarly, we noted the rapid evolution of Fluozin-3fluorescence in neurons following $30 \mathrm{~min}$-exposures to the hypoxia-inducing chemicals $\mathrm{CoCl}_{2}$ ( $1 \mathrm{mM}$; Figures 1D,M), DFX ( $3 \mathrm{mM}$; Figures 1E,M), and $\mathrm{NaN}_{3}(2 \mathrm{mM}$; Figures 1F,M). Three hours later, the intensity of the intracellular fluorescence was significantly reduced in the hypoxic chemical-treated neurons (Figures 1G-I,M). These results thus indicate that $\mathrm{Zn}^{2+}$ was released and accumulated in neurons shortly after exposure to hypoxic chemicals, and thereafter gradually disappears as the time progresses.

\section{INTRACELLULAR ZN ${ }^{2+}$ RELEASE/ACCUMULATION PRECEDES NEURONAL DEATH DURING CHEMICAL HYPOXIA}

We assessed neuronal death for $24 \mathrm{~h}$ after hypoxic insult. On the basis of PI exclusion assay, we found that neurons were still intact at $30 \mathrm{~min}$ after $\mathrm{ZnCl}_{2}$ - or hypoxic chemical treatment (Figure 2), when $\mathrm{Zn}^{2+}$ had highly accumulated in neurons (Figure 1). However, neuronal death began to appear about 3 h later (Figure 3) when intracellular $\mathrm{Zn}^{2+}$-fluorescence decreased (Figure 1), and gradually increased as time progressed, a phenomenon that was further evidenced by MTT cell viability assay (Figure 3). Hence, these data indicate that intracellular $\mathrm{Zn}^{2+}$ release/accumulation precedes neuronal death after chemicalinduced hypoxia, thus providing the $\mathrm{Zn}^{2+}$-induced delayed neuronal death.

\section{EFFECTS OF ZN ${ }^{2+}$ CHELATION ON CHEMICAL HYPOXIA-INDUCED NEURONAL DEATH}

To relieve intracellular $\mathrm{Zn}^{2+}$ overload, we added CaEDTA or ZnEDTA (each $1 \mathrm{mM}$ ) to the media at $10 \mathrm{~min}$ after exposure
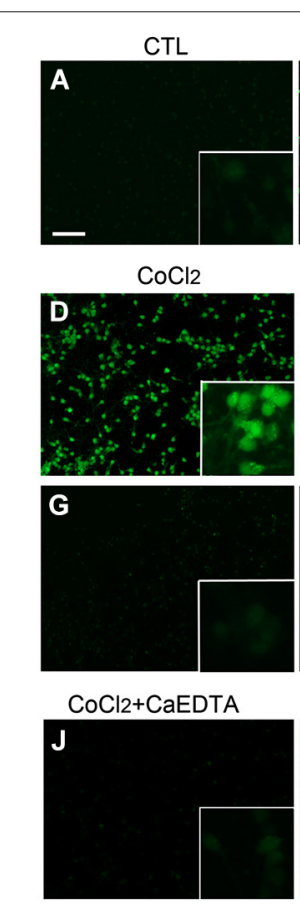

M

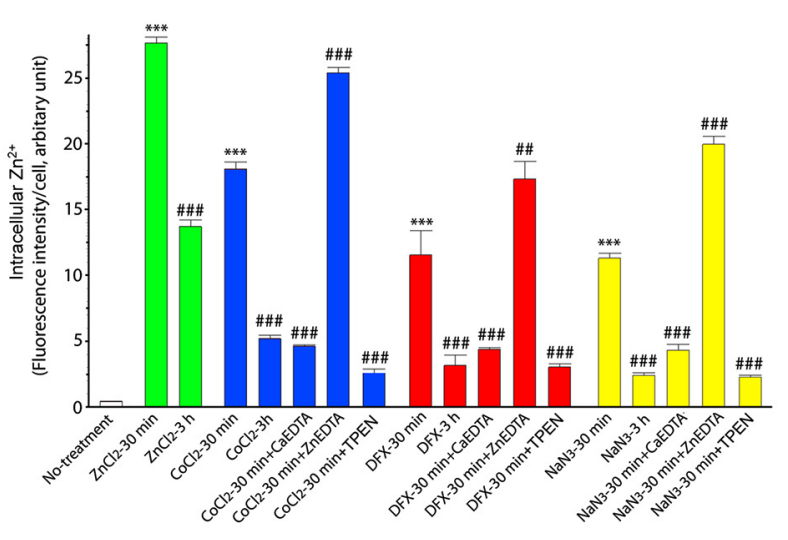

FIGURE 1 | Intracellular $\mathrm{Zn}^{2+}$ release/accumulation in neurons exposed to hypoxia-inducing chemicals. At $30 \mathrm{~min}$ (A,B,D-F) or $3 \mathrm{~h}(\mathbf{C}, \mathbf{G}-\mathbf{I})$ after treatment with $200 \mu \mathrm{M} \mathrm{ZnCl}_{2}$ (B,C), $1 \mathrm{mM} \mathrm{CoCl}_{2}$ (D,G), $3 \mathrm{mM} \mathrm{DFX}$ $(\mathbf{E}, \mathbf{H})$, or $2 \mathrm{mM} \mathrm{NaN}_{3}(\mathbf{F}, \mathbf{I})$, cultured neurons were incubated with $2 \mu \mathrm{M}$ FluoZin-3 AM for $30 \mathrm{~min}$. $\mathrm{CoCl}_{2}$-exposed neurons were also added with $1 \mathrm{mM}$ CaEDTA (J), 1 mM ZnEDTA (K) or TPEN (0.5 $\mu$ M; L) 10 min later. Insets show highly magnified FluoZin-3-stained neurons. Scale bar, $100 \mu \mathrm{m}$. The intensity of FluoZin-3 fluorescence was quantified in neuronal cultures under a variety of chemical combinations (M). Bars denote the mean intensity of the intracellular $\mathrm{Zn}^{2+}$ fluorescence per neuron after subtracting the background level taken from the cell-free area. All experiments were performed in four independent replications $(n=4)$, and the intensity of FluoZin-3 fluorescence was quantified by measuring 3 random spots per each independent sample and expressed as arbitrary units. Data are the mean \pm SEM of quadruplicate experiments. ${ }^{\# \#} p<0.01$, or ${ }^{* *}$ or ${ }^{\# \#} p<0.001$ in comparison with the corresponding control according to one-way ANOVA followed by the Student-Newman-Keuls post hoc test.

of the neurons to $\mathrm{ZnCl}_{2}$ or hypoxic chemicals, and examined FluoZin-3-fluorescence $30 \mathrm{~min}$ later. Consistent with our 


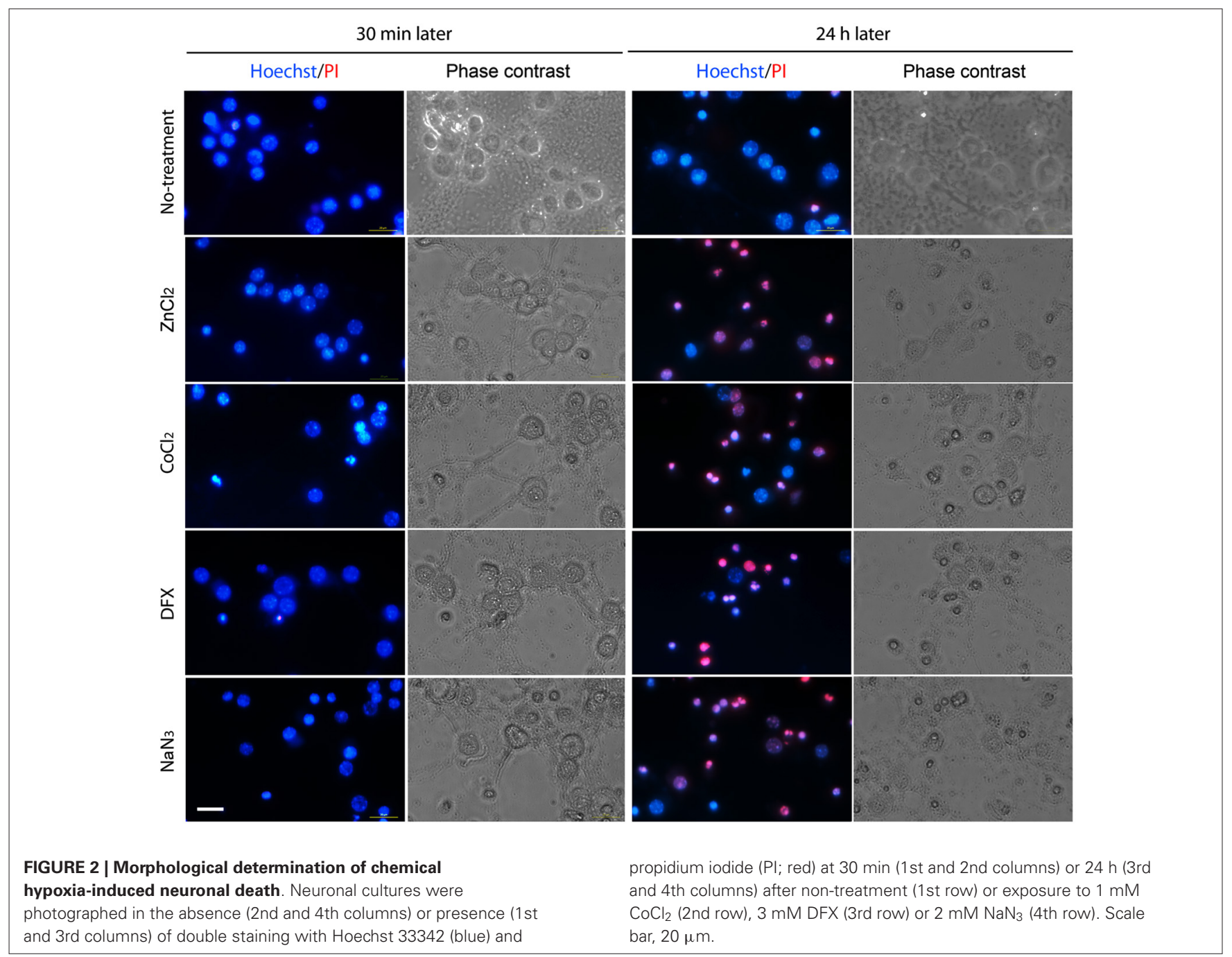

expectations that EDTA would fully chelate intracellular $\mathrm{Zn}^{2+}$ (Frederickson et al., 2002), CaEDTA reduced FluoZin3fluorescence in the $\mathrm{ZnCl}_{2}$ - or hypoxic chemical-exposed neurons (Figure 1) whereas ZnEDTA increased fluorescence (Figures 1K,M). Furthermore, TPEN $(0.5 \mu \mathrm{M})$ perfectly depleted it (Figures 1L,M).

Previous studies using various experimental models of neurological disease have reported that CaEDTA (but not ZnEDTA) efficiently blocks neuronal death (Koh et al., 1996). Hence, in order to define the $\mathrm{Zn}^{2+}$-specific actions on chemical hypoxia-induced neuronal death, we added various salt forms of EDTA $(0.1-1.0 \mathrm{mM})$ or TPEN $(0.1-0.5 \mu \mathrm{M})$ to the culture media at $10 \mathrm{~min}$ or $3 \mathrm{~h}$ after exposure to $\mathrm{ZnCl}_{2}$ or a hypoxic chemical, and then determined cell viability using the MTT assay (Figures 4-7).

In consistency with the previous studies (Koh et al., 1996), either CaEDTA (0.3-1.0 mM) or TPEN (0.1-0.5 $\mu \mathrm{M})$ evidently reduced neuronal death when added at $10 \mathrm{~min}$ after the $\mathrm{ZnCl}_{2}$-exposure (Figure $4 \mathrm{~A}$ ), but the neuroprotective effect was less evident with the 3 h-delayed CaEDTA treatment
(Figure 4B). In contrast, ZnEDTA $(0.1$ or $1.0 \mathrm{mM})$ aggravated the $\mathrm{ZnCl}_{2}$-induced neuronal death (Figures 4A,B). These results support the evidence for $\mathrm{Zn}^{2+}$-induced delayed neuronal death.

When either CaEDTA $(1 \mathrm{mM})$ or TPEN $(0.5 \mu \mathrm{M})$ was added to $\mathrm{CoCl}_{2}$-exposed neurons $10 \mathrm{~min}$ later, it significantly decreased neuronal death; however, ZnEDTA (1 mM) resulted in severe toxicity, and CoEDTA $(1 \mathrm{mM})$ or FeEDTA $(1 \mathrm{mM})$ had no effect (Figure 5A). Three hour post-treatment of CaEDTA or TPEN rarely affected $\mathrm{CoCl}_{2}$-induced neuronal death (Figure 5B). These findings suggest that intracellular $\mathrm{Zn}^{2+}$ can cause delayed neuronal death during $\mathrm{CoCl}_{2}$-induced hypoxia.

However, it was unexpected that ZnEDTA reduced the DFXor $\mathrm{NaN}_{3}$-induced neuronal death when added $10 \mathrm{~min}$ later, but neither CaEDTA nor TPEN (Figures 6A, 7A). FeEDTA also provided some protective effects against DFX-induced hypoxic death (Figure 6A). Three hour post-treatment of EDTA or TPEN had no effect on NaN3 (Figure 6B)- or DFX (Figure 7B)-induced neuronal death. 
A

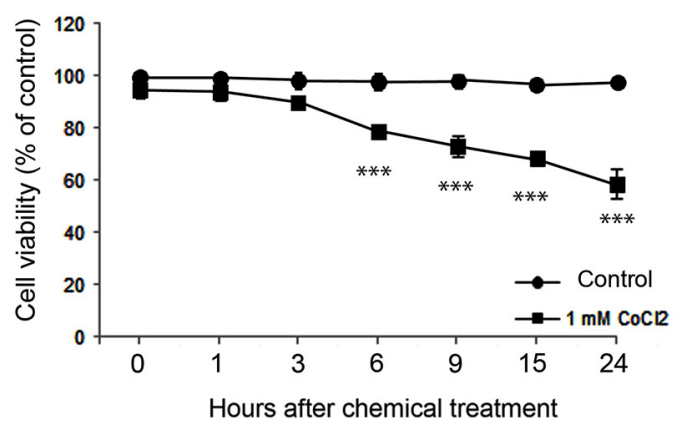

B

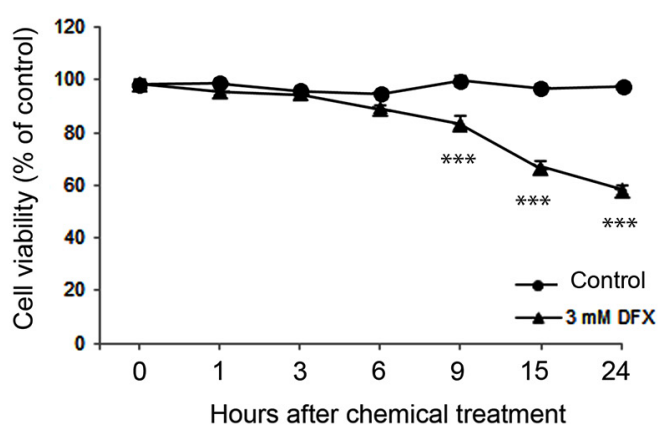

C

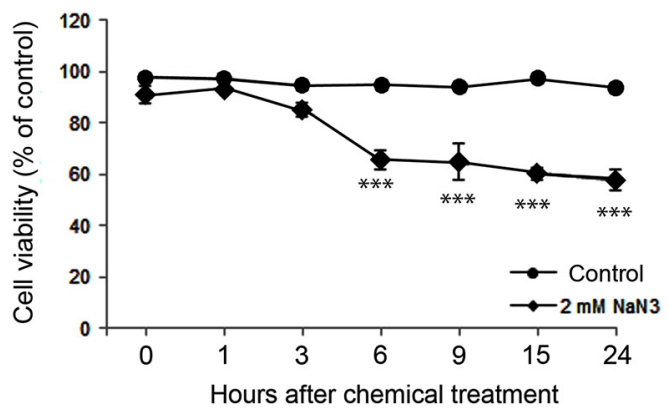

FIGURE 3 | Time course of neuronal death assessed using the MTT cell viability assay at various time points after $1 \mathrm{mM} \mathrm{CoCl} \mathbf{C}_{2}$ (A), $3 \mathrm{mM}$ DFX(B), or $2 \mathrm{mM} \mathrm{NaN}_{3}$ - (C) treatment. Data are the mean \pm SEM of quadruplicate independent experiments, which contained three parallel cultures. ${ }^{* *} p<0.001$ in comparison with the corresponding control treatment according to one-way ANOVA followed by the Student-Newman-Keuls post hoc test.

\section{NEUROPROTECTIVE EFFECT OF METALS IN CHEMICAL HYPOXIA}

Because ZnEDTA prominently reduced neuronal death following DFX- or $\mathrm{NaN}_{3}$-induced hypoxia (Figures 6A, 7A), we investigated whether $\mathrm{Zn}^{2+}$ enables neurons to survive the chemical-induced hypoxic damage (Figures 5C, 6C, 7C). As expected, $\mathrm{ZnCl}_{2}$ $(0.05-1.0 \mathrm{mM})$ significantly increased the level of $\mathrm{CoCl}_{2}-$ induced neuronal death (Figure 5C). However, $\mathrm{ZnCl}_{2}$ produced protective effects against neuronal death following DFX- or $\mathrm{NaN}_{3}$-induced chemical hypoxia (Figures 6C, 7C). Similar to the neuroprotection by FeEDTA against DFX-induced neuronal death (Figure 6A), supplementation with iron $(0.3-1.0 \mathrm{mM}$ $\mathrm{FeCl}_{2}$ or $\mathrm{FeCl}_{3}$ ) rendered neurons significantly more resistant to DFX-induced chemical hypoxia (Figure 6C). Therefore, apart from $\mathrm{CoCl}_{2}$-induced neuronal death that was aggravated by $\mathrm{ZnCl}_{2}, \mathrm{Zn}^{2+}$ is likely to protect neurons against DFX- or $\mathrm{NaN}_{3}-$ induced chemical hypoxia. Plus, iron $\left(\mathrm{Fe}^{2+}\right.$ or $\left.\mathrm{Fe}^{3+}\right)$ may also provide neuroprotective effects against DFX-induced hypoxia (Figure 6C).

\section{DISCUSSION}

The mechanism underlying chemical hypoxia remains unclear. A line of studies have noted to the involvement of iron in stabilizing HIFl $\alpha$ and thereby activating hypoxic signals (Ho and Bunn, 1996). Because HIF1 $\alpha$ is rapidly degraded by the polyubiquitination and proteasome pathway, which is manipulated by prolyl-4-hydroxylases (PHDs), it is normally present in cells at low levels (Bruick and McKnight, 2001; Epstein et al., 2001). PHDs essentially require oxygen and iron for their activity, so the depletion of iron from cells could inhibit the activity of the PHDs to stabilize HIF1 $\alpha$ from degradation, stimulating the hypoxic responses similar to that observed due to an oxygen shortage (Bruick and McKnight, 2001; Guo et al., 2001). Transition metals (e.g., $\mathrm{Co}^{2+}$ or $\mathrm{Ni}^{2+}$ ) and iron chelators (e.g., DFX) could induce hypoxic responses by inhibiting PHD activity via iron replacement or depletion, respectively (Schofield and Ratcliffe, 2004; Choi et al., 2006). Although $\mathrm{Zn}^{2+}$ could be another effective replacement metal for iron in PHDs (Shibayama et al., 1986), there have been disputes regarding the roles of $\mathrm{Zn}^{2+}$ in hypoxia. $\mathrm{Zn}^{2+}$ has recently been found to elevate the intracellular expression of HIF $1 \alpha$ through the activation of NADPH oxidase or poly(ADP ribose) polymerase (PARP; Pan et al., 2013; Malairaman et al., 2014). By contrast, $\mathrm{Zn}^{2+}$ also inhibits HIFl $\alpha$ activity and the activation of the hypoxia-inducible genes to block the hypoxic responses (Chun et al., 2000, 2001). Thus, while these HIF1 $\alpha$-modulating metal signals may suggest a mechanism of chemical hypoxia, it still remains to be defined how hypoxic chemicals induce neuron death, particularly via intracellular $\mathrm{Zn}^{2+}$ release/accumulation.

In this study, when the neuronal cultures were exposed to $\mathrm{ZnCl}_{2}$, or the hypoxic chemical $\mathrm{CoCl}_{2}, \mathrm{DFX}$, or $\mathrm{NaN}_{3}$, we observed the intense emission of $\mathrm{Zn}^{2+}$-specific FluoZin3 -fluorescence in neurons. To confirm the intracellular $\mathrm{Zn}^{2+}$ release/accumulation, we examined that the $\mathrm{Zn}^{2+}$-chelator CaEDTA (Koh et al., 1996; Frederickson et al., 2002) evidently eliminated FluoZin-3-fluorescence from the chemical-treated cultures at the higher concentration $(1 \mathrm{mM})$, despite concern that low concentration of CaEDTA perturb no response of FluoZin-3 to $\mathrm{Zn}^{2+}$ (Zhao et al., 2008). Moreover, TPEN (0.5 $\mu \mathrm{M})$ also perfectly depleted FluoZin-3-fluorescence, but the non$\mathrm{Zn}^{2+}$ chelator ZnEDTA (1 mM) (Koh et al., 1996) showed no attenuation of the fluorescence intensity. Therefore, these findings support that $\mathrm{Zn}^{2+}$ is robustly released and accumulated in cultured neurons shortly after the hypoxic chemical treatment. A variety of sources of releasable $\mathrm{Zn}^{2+}$ has been found in neurons, such as $\mathrm{Zn}^{2+}$-bound proteins (Aizenman et al., 2000; Lee et al., 2000, 2003) or $\mathrm{Zn}^{2+}$-containing organelles including mitochondria (Jiang et al., 2001; Sensi et al., 2002) or lysosomes (Hwang et al., 2008). In addition, since neurons survived the moment of the highest intracellular $\mathrm{Zn}^{2+}$ accumulation and then 
A

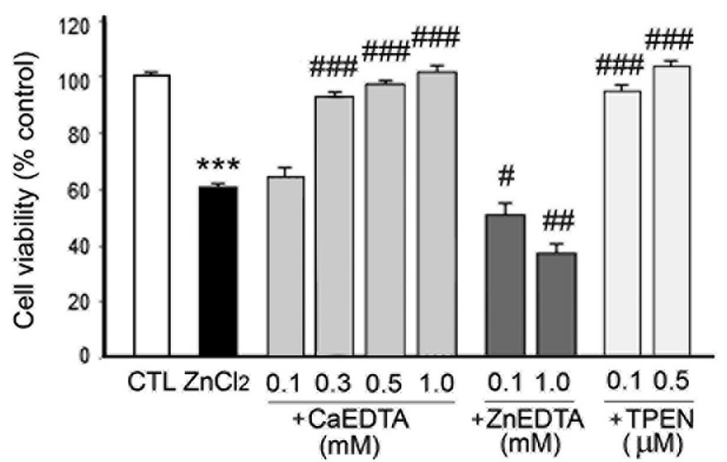

FIGURE 4 | Effects of metal chelation on $\mathrm{ZnCl}_{2}$-induced neuron death. $\mathrm{ZnCl}_{2}(200 \mu \mathrm{M})$-exposed neurons were followed by the immediate (A) or $3 \mathrm{~h}$ later (B) addition of CaEDTA (0.1-1.0 mM), ZnEDTA (0.1 or $1.0 \mathrm{mM})$, or TPEN (0.1 or $0.5 \mu \mathrm{M})$ ). Twenty-four hours after $\mathrm{ZnCl}_{2}$ application, neuronal death was assessed by the MTT cell viability assay. Bars denote the

\section{B}

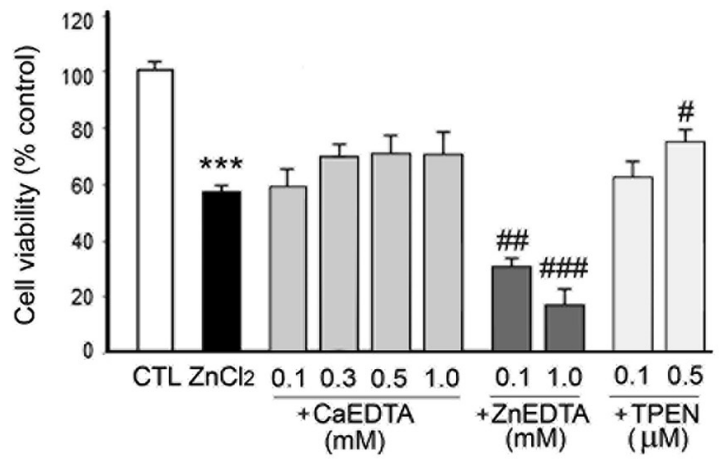

mean \pm SEM of at least three independent experiments, which each consisted of three parallel cultures. Values were expressed as percentages of non-treated control cells. ${ }^{\#} p<0.05$, ${ }^{\# \#} p<0.01$, or ${ }^{* * *}$ or ${ }^{\# \#} p<0.001$ in the corresponding comparison according to one-way ANOVA followed by the Student-Newman-Keuls post hoc test.

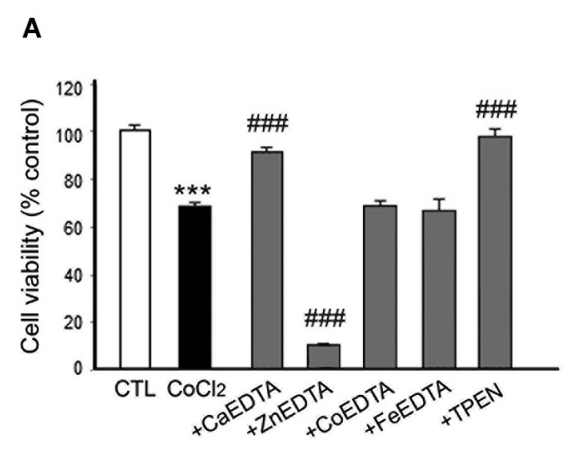

B

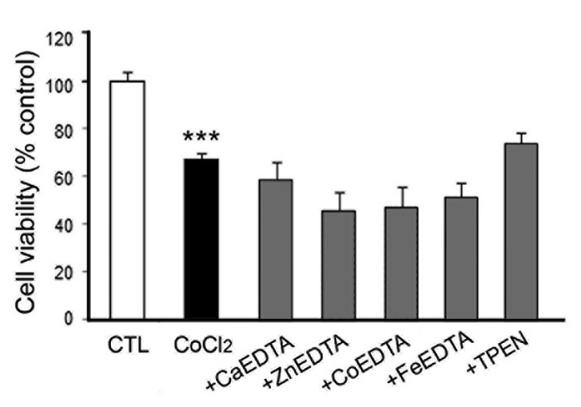

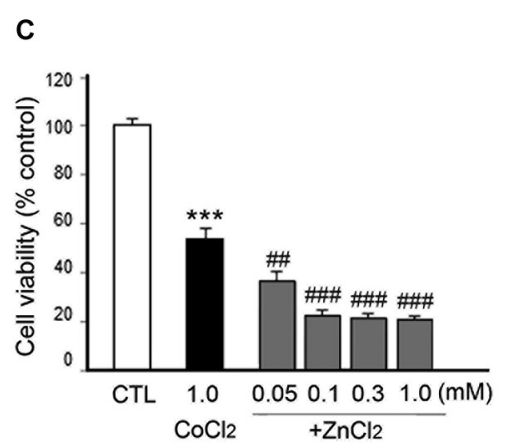

FIGURE 5 | Effects of metal chelation or $\mathrm{Zn}^{2+}$ supplementation on $\mathrm{CoCl}_{2}$-induced neuronal death. $\mathrm{CoCl}_{2}(1 \mathrm{mM})$-treated neurons were followed by the immediate (A) or $3 \mathrm{~h}$ later (B) addition of CaEDTA (1.0 mM), ZnEDTA (1.0 mM), CoEDTA (1.0 mM), FeEDTA (1.0 mM), or TPEN $(0.5 \mu \mathrm{M})$, or by the immediate addition of $\mathrm{ZnCl}_{2}(0.05-1.0 \mathrm{mM})$ (C). Twenty-four hours after $\mathrm{CoCl}_{2}$ application, neuronal death was assessed using the MTT cell viability assay. Bars denote the mean \pm SEM of at least three independent experiments, which each consisted of three parallel cultures. Values were expressed as percentages of non-treated control cells. ${ }^{\# \#} p<0.01$, or ${ }^{* *}$ or \#\#\# $p<0.001$ in the corresponding comparison by the one-way ANOVA and the Student-Newman-Keuls post hoc test. started to die along with its gradual loss, we guess that $\mathrm{Zn}^{2+}$ could cause delayed neuronal death in hypoxic chemical-treated cultures.

However, the effects of $\mathrm{Zn}^{2+}$ chelation on chemical hypoxiainduced neuronal death differed depending on the hypoxic chemical that was used. When EDTA was added immediately after $\mathrm{CoCl}_{2}$-induced hypoxia, CaEDTA evidently alleviated neuron death, but ZnEDTA potently augmented cell death. However, $3 \mathrm{~h}$ delayed CaEDTA rarely reduced $\mathrm{CoCl}_{2}$-induced neuronal death. CoEDTA or FeEDTA had no effects. A strong intracellular $\mathrm{Zn}^{2+}$-chelator TPEN also produced the neuroprotective effects. These results were comparable to the effect of CaEDTA or TPEN on $\mathrm{ZnCl}_{2}$-induced delayed neuronal death, where the immediate $\mathrm{Zn}^{2+}$ chelation with CaEDTA or TPEN counteracted the neuronal death but the late CaEDTA showed no protection. It appears that the late $\mathrm{Zn}^{2+}$ chelation couldn't afford to block the death signaling process that has been already triggered by the precedent $\mathrm{Zn}^{2+}$ overload in neurons. Therefore, we believe that $\mathrm{CoCl}_{2}$-induced hypoxia rapidly triggers intracellular $\mathrm{Zn}^{2+}$ release, leading to $\mathrm{Zn}^{2+}$ overload in neurons and thereby causing their death. In contrast, there was an opposite case during DFX- or $\mathrm{NaN}_{3}$-induced hypoxia. ZnEDTA rather protected neurons from DFX- and $\mathrm{NaN}_{3}$-induced hypoxic death, but CaEDTA had no effect. $\mathrm{Zn}^{2+}$ supplementation also enabled neurons to survive DFX- or $\mathrm{NaN}_{3}-$ induced hypoxic damages. These results suggest that $\mathrm{Zn}^{2+}$ may be neurotoxic or neuroprotective in neurons during chemical hypoxia; $\mathrm{Zn}^{2+}$ may directly cause hypoxic neuronal death (in $\mathrm{CoCl}_{2}$-induced hypoxia), or normally participate in neuronal survival or viability (in DFX- or $\mathrm{NaN}_{3}$-induced hypoxia). In addition, we found that iron supplementation $\left(\mathrm{Fe}^{2+}\right.$ or $\left.\mathrm{Fe}^{3+}\right)$ can protect neurons from DFX-induced hypoxic damage, consistent 

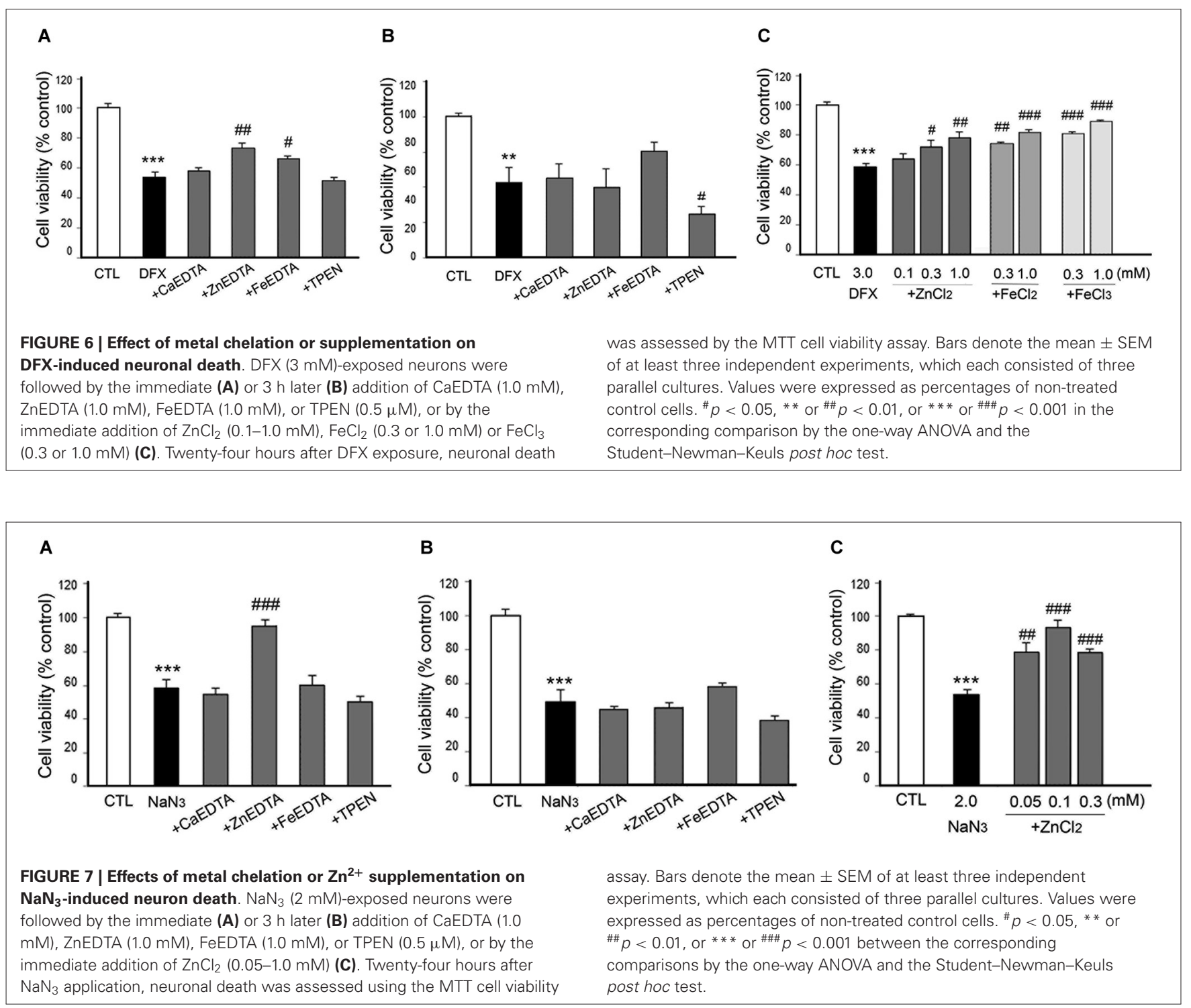

with speculation that it may make up for DFX-induced iron depletion. However, it is unfortunate that there is no current explanation or information concerning how or why $\mathrm{Zn}^{2+}$ plays in the opposite roles in the chemical hypoxia-induced neuronal death.

It is well established that cytosolic calcium $\left(\mathrm{Ca}^{2+}\right)$ overload triggers signal pathways to execute neuronal degeneration after hypoxic/ischemic insult (Lipton, 1999; Bano and Nicotera, 2007; Mattson, 2007; Berna-Erro et al., 2009). Our results might exclude the causative roles of $\mathrm{Ca}^{2+}$ in $\mathrm{CoCl}_{2}$-induced hypoxic neuronal death due to the evidence consistent with the earlier study (Koh et al., 1996), in which cytosolic $\mathrm{Zn}^{2+}$ overload preceded neuronal death and $\mathrm{Zn}^{2+}$-specific chelation CaEDTA (but not ZnEDTA) recovered it. Instead, as we failed to determine whether $\mathrm{Zn}^{2+}$-specific chelation inhibits neuronal death after DFX- or $\mathrm{NaN}_{3}$-inducrd hypoxia, we couldn't rule out the possibility that $\mathrm{Ca}^{2+}$-induced excitotoxicity may contribute to hypoxic neuronal death (Koh et al., 1996; Lipton, 1999; Bano and Nicotera, 2007; Mattson, 2007; Berna-Erro et al., 2009).

In conclusion, we for the first time provide evidence that hypoxia stimulates the intracellular release/accumulation of $\mathrm{Zn}^{2+}$ in neurons, and thereby it may contribute to neuronal death or survival. The opposite roles of $\mathrm{Zn}^{2+}$ in hypoxic chemicalinduced neuron death may not only indicate that different hypoxic chemicals induce neuron death via distinct mechanisms, but reflect the diverse groups of signals that essentially require $\mathrm{Zn}^{2+}$ for their functions. Otherwise, $\mathrm{Zn}^{2+}$-regulated neuronal fate may be differentially determined depending on the actual range of intracellular $\mathrm{Zn}^{2+}$ levels (Cho et al., 2010). To date, chelation study using EDTA or TPEN has focused mainly on the negative roles of $\mathrm{Zn}^{2+}$ as a main cause of neuronal death in the context of excitotoxic acute brain injury (Sensi et al., 2011). Instead, this study offers 
the insight into the positive aspect of $\mathrm{Zn}^{2+}$ that it could mediate neuronal survival under such neurological diseases. Further study will be warranted to elucidate the mechanism by which $\mathrm{Zn}^{2+}$ enable neurons to survive a variety of neurotoxic circumstances.

\section{AUTHOR CONTRIBUTIONS}

Sujeong Kim and Jung-Woo Seo designed the culture experiments and performed the MTT viability analysis. Shin Bi Oh and So Hee Kim photographed the cultured neurons and performed the image-analysis. Inki Kim and Nayoung Suh managed and discussed the overall study, analyzed the data and prepared the manuscript draft. Joo-Yong Lee conceived and designed the work, approved the data analysis and interpretations, and finally completed the manuscript. All authors saw and approved the completion of the work.

\section{ACKNOWLEDGMENTS}

This study was supported by the Korea Healthcare Technology R\&D Project, Ministry for Health and Welfare, Republic of Korea (A092042 to Joo-Yong Lee); the Basic Science Research Program, National Research Foundation of Korea, Ministry of Education, Republic of Korea (NRF-2012R1A1A2006801 to Joo-Yong Lee); and the Asan Institute for Life Sciences, Asan Medical Center, Republic of Korea (2015-396 to Joo-Yong Lee).

\section{REFERENCES}

Aizenman, E., Stout, A. K., Hartnett, K. A., Dineley, K. E., McLaughlin, B., and Reynolds, I. J. (2000). Induction of neuronal apoptosis by thiol oxidation: putative role of intracellular zinc release. J. Neurochem. 75, 1878-1888. doi: 10. 1046/j.1471-4159.2000.0751878.x

Almli, L. M., Hamrick, S. E., Koshy, A. A., Täuber, M. G., and Ferriero, D. M. (2001). Multiple pathways of neuroprotection against oxidative stress and excitotoxic injury in immature primary hippocampal neurons. Brain Res. Dev. Brain Res. 132, 121-129. doi: 10.1016/s0165-3806(01)00302-9

Bano, D., and Nicotera, P. (2007). $\mathrm{Ca}^{2+}$ signals and neuronal death in brain ischemia. Stroke 38, 674-676. doi: 10.1161/01.str.0000256294. 46009.29

Berna-Erro, A., Braun, A., Kraft, R., Kleinschnitz, C., Schuhmann, M. K., Stegner, D., et al. (2009). STIM2 regulates capacitive $\mathrm{Ca}^{2+}$ entry in neurons and plays a key role in hypoxic neuronal cell death. Sci. Signal. 2:ra67. doi: 10.1126/scisignal. 2000522

Bossy-Wetzel, E., Talantova, M. V., Lee, W. D., Schölzke, M. N., Harrop, A., Mathews, E., et al. (2004). Crosstalk between nitric oxide and zinc pathways to neuronal cell death involving mitochondrial dysfunction and $\mathrm{p} 38$-activated $\mathrm{K}^{+}$ channels. Neuron 41, 351-365. doi: 10.1016/s0896-6273(04)00015-7

Bruick, R. K., and McKnight, S. L. (2001). A conserved family of prolyl-4hydroxylases that modify HIF. Science 294, 1337-1340. doi: 10.1126/science. 1066373

Büchner, M., Huber, R., and Riepe, M. W. (2002). Trans-synaptic increase of hypoxic tolerance in hippocampus upon physical challenge with two-photon microscopy. Hippocampus 12, 765-773. doi: 10.1002/hipo.10028

Cho, E., Hwang, J. J., Han, S. H., Chung, S. J., Koh, J. Y., and Lee, J. Y. (2010). Endogenous zinc mediates apoptotic programmed cell death in the developing brain. Neurotox. Res. 17, 156-166. doi: 10.1007/s12640-009-9085-2

Choi, S. M., Choi, K. O., Park, Y. K., Cho, H., Yang, E. G., and Park, H. (2006). Clioquinol, a $\mathrm{Cu}(\mathrm{II}) / \mathrm{Zn}$ (II) chelator, inhibits both ubiquitination and asparagine hydroxylation of hypoxia-inducible factor-lalpha, leading to expression of vascular endothelial growth factor and erythropoietin in normoxic cells. J. Biol. Chem. 281, 34056-34063. doi: 10.1074/jbc.m603 913200

Choi, D. W., Yokoyama, M., and Koh, J. (1988). Zinc neurotoxicity in cortical cell culture. Neuroscience 24, 67-79. doi: 10.1016/0306-4522(88)90312-0
Chun, Y. S., Choi, E., Kim, G. T., Lee, M. J., Lee, M. J., Lee, S. E., et al. (2000). Zinc induces the accumulation of hypoxia-inducible factor (HIF)- $1 \alpha$, but inhibits the nuclear translocation of HIF-1 $\beta$, causing HIF-1 inactivation. Biochem. Biophys. Res. Commun. 268, 652-656. doi: 10.1006/bbrc.2000.2180

Chun, Y. S., Choi, E., Yeo, E. J., Lee, J. H., Kim, M. S., and Park, J. W. (2001). A new HIF-1 alpha variant induced by zinc ion suppresses HIF-1-mediated hypoxic responses. J. Cell Sci. 114, 4051-4061.

Epstein, A. C., Gleadle, J. M., McNeill, L. A., Hewitson, K. S., O’Rourke, J., Mole, D. R., et al. (2001). C. elegans EGL-9 and mammalian homologs define a family of dioxygenases that regulate HIF by prolyl hydroxylation. Cell 107, 43-54. doi: 10.1016/S0092-8674(01)00507-4

Fang, D., Li, Z., Zhong-Ming, Q., Mei, W. X., Ho, Y. W., Yuan, X. W., et al. (2008). Expression of bystin in reactive astrocytes induced by ischemia/reperfusion and chemical hypoxia in vitro. Biochim. Biophys. Acta 1782, 658-663. doi: 10.1016/j. bbadis.2008.09.007

Frederickson, C. J., Hernandez, M. D., Goik, S. A., Morton, J. D., and McGinty, J. F. (1988). Loss of zinc staining from hippocampal mossy fibers during kainic acid induced seizures: a histofluorescence study. Brain Res. 446, 383-386. doi: 10. 1016/0006-8993(88)90899-2

Frederickson, C. J., Suh, S. W., Koh, J. Y., Cha, Y. K., Thompson, R. B., LaBuda, C. J., et al. (2002). Depletion of intracellular zinc from neurons by use of an extracellular chelator in vivo and in vitro. J. Histochem. Cytochem. 50, 16591662. doi: 10.1177/002215540205001210

Garnier, P., Ying, W., and Swanson, R. A. (2003). Ischemic preconditioning by caspase cleavage of poly(ADP-ribose) polymerase-1. J. Neurosci. 23, 7967-7973.

Gee, K. R., Zhou, Z. L., Ton-That, D., Sensi, S. L., and Weiss, J. H. (2002). Measuring zinc in living cells. A new generation of sensitive and selective fluorescent probes. Cell Calcium 31, 245-251. doi: 10.1016/S0143-4160(02) 00053-2

Guelman, L. R., Pagotto, R. M., Di Toro, C. G., and Zieher, L. M. (2004). Deferoxamine antioxidant activity on cerebellar granule cells gammairradiated in vitro. Neurotoxicol. Teratol. 26, 477-483. doi: 10.1016/j.ntt. 2004.02.001

Guo, K., Searfoss, G., Krolikowski, D., Pagnoni, M., Franks, C., Clark, K., et al. (2001). Hypoxia induces the expression of the pro-apoptotic gene BNIP3. Cell Death Differ. 8, 367-376. doi: 10.1038/sj.cdd.4400810

Ho, V. T., and Bunn, H. F. (1996). Effects of transition metals on the expression of the erythropoietin gene: further evidence that the oxygen sensor is a heme protein. Biochem. Biophys. Res. Commun. 223, 175-180. doi: 10.1006/bbrc.1996. 0865

Hwang, J. J., Lee, S. J., Kim, T. Y., Cho, J. H., and Koh, J. Y. (2008). Zinc and 4hydroxy-2-nonenal mediate lysosomal membrane permeabilization induced by $\mathrm{H} 2 \mathrm{O} 2$ in cultured hippocampal neurons. J. Neurosci. 28, 3114-3122. doi: 10. 1523/JNEUROSCI.0199-08.2008

Jiang, D., Sullivan, P. G., Sensi, S. L., Steward, O., and Weiss, J. H. (2001). Zn ${ }^{2+}$ induces permeability transition pore opening and release of pro-apoptotic peptides from neuronal mitochondria. J. Biol. Chem. 276, 47524-47529. doi: 10. 1074/jbc.m108834200

Koh, J. Y., Suh, S. W., Gwag, B. J., He, Y. Y., Hsu, C. Y., and Choi, D. W. (1996). The role of zinc in selective neuronal death after transient global cerebral ischemia. Science 272, 1013-1016. doi: 10.1126/science.272.5264.1013

Lee, J. Y., Cole, T. B., Palmiter, R. D., and Koh, J. Y. (2000). Accumulation of zinc in degenerating hippocampal neurons of ZnT3-null mice after seizures: evidence against synaptic vesicle origin. J. Neurosci. 20:RC79.

Lee, J. Y., Kim, J. H., Palmiter, R. D., and Koh, J. Y. (2003). Zinc released from metallothionein-iii may contribute to hippocampal CA1 and thalamic neuronal death following acute brain injury. Exp. Neurol. 184, 337-347. doi: 10. 1016/s0014-4886(03)00382-0

Lipton, P. (1999). Ischemic cell death in brain neurons. Physiol. Rev. 79, 1431-1568.

Malairaman, U., Dandapani, K., and Katyal, A. (2014). Effect of $\mathrm{Ca}_{2}$ EDTA on zinc mediated inflammation and neuronal apoptosis in hippocampus of an in vivo mouse model of hypobaric hypoxia. PLoS One 9:e110253. doi: 10.1371/journal. pone. 0110253

Mattson, M. P. (2007). Calcium and neurodegeneration. Aging Cell 6, 337-350. doi: 10.1111/j.1474-9726.2007.00275.x

Medvedeva, Y. V., Lin, B., Shuttleworth, C. W., and Weiss, J. H. (2009). Intracellular $\mathrm{Zn}^{2+}$ accumulation contributes to synaptic failure, mitochondrial depolarization and cell death in an acute slice oxygen-glucose deprivation model 
of ischemia. J. Neurosci. 29, 1105-1114. doi: 10.1523/JNEUROSCI.4604-08. 2009

Miyawaki, T., Yokota, H., Oguro, K., Kato, K., and Shimazaki, K. (2004). Ischemic preconditioning decreases intracellular zinc accumulation induced by oxygen-glucose deprivation in gerbil hippocampal CA1 neurons. Neurosci. Lett. 362, 216-219. doi: 10.1016/s0304-3940(04) 00326-x

Pan, R., Chen, C., Liu, W. L., and Liu, K. J. (2013). Zinc promotes the death of hypoxic astrocytes by upregulating hypoxia-induced hypoxia-inducible factor-1alpha expression via poly(ADP-ribose) polymerase-1. CNS Neurosci. Ther. 19, 511-520. doi: 10.1111/cns.12098

Roemgens, A., Singh, S., Beyer, C., and Arnold, S. (2011). Inducers of chemical hypoxia act in a gender- and brain region-specific manner on primary astrocyte viability and cytochrome C oxidase. Neurotox. Res. 20, 1-14. doi: 10. 1007/s12640-010-9213-z

Schofield, C. J., and Ratcliffe, P. J. (2004). Oxygen sensing by HIF hydroxylases. Nat. Rev. Mol. Cell Biol. 5, 343-354. doi: 10.1038/nrm1366

Selvatici, R., Previati, M., Marino, S., Marani, L., Falzarano, S., Lanzoni, I., et al. (2009). Sodium azide induced neuronal damage in vitro: evidence for nonapoptotic cell death. Neurochem. Res. 34, 909-916. doi: 10.1007/s11064-0089852-0

Sensi, S. L., Paoletti, P., Koh, J. Y., Aizenman, E., Bush, A. I., and Hershfinkel, M. (2011). The neurophysiology and pathology of brain zinc. J. Neurosci. 31, 16076-16085. doi: 10.1523/JNEUROSCI.3454-11.2011

Sensi, S. L., Ton-That, D., and Weiss, J. H. (2002). Mitochondrial sequestration and $\mathrm{Ca}^{2+}$-dependent release of cytosolic $\mathrm{Zn}^{2+}$ loads in cortical neurons. Neurobiol. Dis. 10, 100-108. doi: 10.1006/nbdi.2002.0493

Sharp, F. R., and Bernaudin, M. (2004). HIF1 and oxygen sensing in the brain. Nat. Rev. Neurosci. 5, 437-448. doi: 10.1038/nrn1408

Shibayama, N., Morimoto, H., and Miyazaki, G. (1986). Oxygen equilibrium study and light absorption spectra of $\mathrm{Ni}(\mathrm{II})$-Fe(II) hybrid hemoglobins. J. Mol. Biol. 192, 323-329. doi: 10.1016/0022-2836(86)90367-0

Stanciu, M., Wang, Y., Kentor, R., Burke, N., Watkins, S., Kress, G., et al. (2000). Persistent activation of ERK contributes to glutamate-induced oxidative toxicity in a neuronal cell line and primary cortical neuron cultures. J. Biol. Chem. 275, 12200-12206. doi: 10.1074/jbc.275.16.12200

Suh, S. W., Chen, J. W., Motamedi, M., Bell, B., Listiak, K., Pons, N. F., et al. (2000). Evidence that synaptically-released zinc contributes to neuronal injury after traumatic brain injury. Brain Res. 852, 268-273. doi: 10.1016/s00068993(99)02095-8
Suh, S. W., Garnier, P., Aoyama, K., Chen, Y., and Swanson, R. A. (2004). Zinc release contributes to hypoglycemia-induced neuronal death. Neurobiol. Dis. 16, 538-545. doi: 10.1016/j.nbd.2004.04.017

Tønder, N., Johansen, F. F., Frederickson, C. J., Zimmer, J., and Diemer, N. H. (1990). Possible role of zinc in the selective degeneration of dentate hilar neurons after cerebral ischemia in the adult rat. Neurosci. Lett. 109, 247-252. doi: 10.1016/0304-3940(90)90002-q

White, A. R., Huang, X., Jobling, M. F., Barrow, C. J., Beyreuther, K., Masters, C. L., et al. (2001). Homocysteine potentiates copper- and amyloid beta peptidemediated toxicity in primary neuronal cultures: possible risk factors in the Alzheimer's-type neurodegenerative pathways. J. Neurochem. 76, 1509-1520. doi: 10.1046/j.1471-4159.2001.00178.x

Yin, H. Z., Sensi, S. L., Ogoshi, F., and Weiss, J. H. (2002). Blockade of $\mathrm{Ca}^{2+}$. permeable AMPA/kainate channels decreases oxygen-glucose deprivationinduced $\mathrm{Zn}^{2+}$ accumulation and neuronal loss in hippocampal pyramidal neurons. J. Neurosci. 22, 1273-1279.

Zhang, S., Chen, X., Yang, Y., Zhou, X., Liu, J., and Ding, F. (2011). Neuroprotection against cobalt chloride-induced cell apoptosis of primary cultured cortical neurons by salidroside. Mol. Cell. Biochem. 354, 161-170. doi: 10.1007/s11010011-0815-4

Zhao, J., Bertoglio, B. A., Gee, K. R., and Kay, A. R. (2008). The zinc indicator FluoZin-3 is not perturbed significantly by physiological levels of calcium or magnesium. Cell Calcium 44, 422-426. doi: 10.1016/j.ceca.2008.01.006

Conflict of Interest Statement: The authors declare that the research was conducted in the absence of any commercial or financial relationships that could be construed as a potential conflict of interest.

Received: 05 November 2014; accepted: 03 January 2015; published online: 23 January 2015.

Citation: Kim S, Seo J-W, Oh SB, Kim SH, Kim I, Suh N and Lee J-Y (2015) Disparate roles of zinc in chemical hypoxia-induced neuronal death. Front. Cell. Neurosci. 9:1. doi: 10.3389/fncel.2015.00001

This article was submitted to the journal Frontiers in Cellular Neuroscience.

Copyright (C) $2015 \mathrm{Kim}$, Seo, Oh, Kim, Kim, Suh and Lee. This is an open-access article distributed under the terms of the Creative Commons Attribution License (CC BY). The use, distribution and reproduction in other forums is permitted, provided the original author(s) or licensor are credited and that the original publication in this journal is cited, in accordance with accepted academic practice. No use, distribution or reproduction is permitted which does not comply with these terms. 